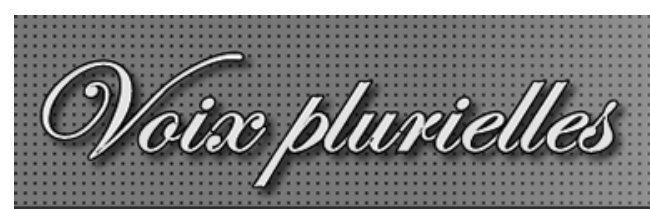

Revue de l'Association des Professeur-e-s de Français des Universités et Collèges Canadiens

\author{
Voix plurielles \\ Volume 4, Numéro 1 : mai 2007
}

\title{
Mariana Ionescu
}

\section{L'ici-là selon Gisèle Pineau}

Citation MLA : Ionescu, Mariana. «L'ici-là selon Gisèle Pineau.» Voix plurielles 4.1 (mai 2007).

(C) Voix plurielles, revue électronique de l'APFUCC 2007. 


\title{
L'ici-là selon Gisèle Pineau
}

\author{
Mariana Ionescu \\ Huron University College
}

Mai 2007

\section{Résumé analytique}

\begin{abstract}
Tet article porte sur la façon dont l'écrivain antillais Gisèle Pineau entrevoit la possibilité de rapprocher des espaces culturels apparemment irréconciliables par le biais d'un nouvel espace, celui de la remémoration et de l'écriture. Dans cet entre-deux, espace métissé par excellence, les personnages féminins de L'Exil selon Julia, ainsi que ceux d'autres écrits (auto) biographiques de Pineau, se confrontent à de nouveaux signes identitaires qui les obligent à jeter un regard critique sur le pays d'accueil, sans pour autant idéaliser à outrance le pays d'origine. Une attention particulière sera attachée au vocable créole ici-là, porteur de significations multiples.
\end{abstract}

\section{Introduction}

« Ecrire en tant que femme noire créole, c'est apporter ma voix aux autres voix des femmes d'ici et d'ailleurs...» (Penser la créolité 289). En effet, depuis son premier roman, La grande drive des esprits (1993), jusqu'à son dernier, Mes quatre femmes (2007), Gisèle Pineau n'a jamais abandonné cette trace de l'écriture engagée, mise au service des femmes. Cependant, même si les personnages féminins constituent la majorité des protagonistes de ses récits, les hommes n'en sont pas exclus, en dépit de tout le malheur que certains leur infligent. En outre, rares sont les récits dans lesquels cet auteur ne rêve d'une humanité sans haine, sans violence et sans racisme. Sa vision du monde dépasse les limites de sa petite île, aussi bien que celles de sa race. " Ecrire en tant que noire », précise-t-elle, «c'est vivre l'espérance d'un monde vraiment nouveau, peuples, races, langues, religions, cultures mêlés, imbriqués, s'enrichissant, se découvrant sans cesse, se respectant et s'acceptant dans la belle différence» (Penser la créolité 295).

\section{Le projet (auto)biographique de Gisèle Pineau}

Il convient tout d'abord de noter qu'à partir de ses expériences d'enfance passée en métropole entre des parents désireux de franciser leurs enfants et une grand-mère déterminée à les préparer pour le retour à l'île, Pineau décide de partager son vécu et surtout de ne rien passer sous silence

Il fallait aussi raconter les histoires de l'exil créole, la situation des 
Antillais dans les banlieues parisiennes, le sentiment d'amour-haine pour la France, le manque du pays d'origine, le rejet des manières créoles, de la langue, des croyances magico-religieuses... (Penser la créolité 291).

A travers le vécu des personnages de ses écrits, elle évoque des espaces multiples, mais qui sont tous plus ou moins imprégnés de la culture insulaire guadeloupéenne. Bien que née en France, cet auteur invite à la réappropriation de l'espace antillais, sans pour autant rejeter les autres lieux qui ont marqué sa construction identitaire, ainsi que celle de ses personnages. Qu'il s'agisse de la Caraïbe francophone et anglophone, de la France, de l'Afrique ou des Etats-Unis, elle explore avec prédilection l'espace de l'exil des Antillais, et surtout des Antillaises, présentées comme réceptacles des souffrances familiales et collectives ${ }^{1}$.

Cette thématique complexe se trouve au cœur de son projet (auto)biographique, qui fera l'objet de cet article. Nous analyserons particulièrement la façon dont Gisèle Pineau entrevoit la possibilité de rapprocher des espaces culturels apparemment irréconciliables par le biais de nouveaux espaces, ceux de la remémoration et de l'écriture. Dans ces entre-deux, espaces métissés par excellence, nombre de ses personnages féminins, particulièrement les plus jeunes, se confrontent à de nouveaux signes identitaires qui les obligent à jeter un regard critique sur le pays d'accueil, sans pour autant idéaliser à outrance le pays d'origine.

La plupart de nos observations porteront sur L'Exil selon Julia (1996), récit à caractère autobiographique dont la narration est assumée par une jeune narratrice, Marie ${ }^{2}$. Nous ferons également des références au roman pour adolescents Un Papillon dans la cité (1992), à L'Âme prêtée aux oiseaux (1998) et à Chair Piment (2002). Une attention particulière sera attachée au déictique créole ici-là qui change de signification en fonction de la réalité extratextuelle à laquelle il renvoie. Qu'il s'agisse du pays d'exil ou du Pays tout court, voire la Guadeloupe, ce vocable rapproche l'ici et l'ailleurs, permettant au lecteur de Pineau de se déplacer constamment d'un espace à l'autre.

\section{L'Exil selon Julia - autofiction?}

Dans une causerie avec Christiane Makward, Gisèle Pineau précise que l'écriture de L'Exil selon Julia l'a aidée à se réconcilier avec son enfance et à s'accepter « en tant qu'être humain » (" Causerie » 225). En effet, dans ce récit il s'agit de la petite enfance de l'auteur, passée tout d'abord dans un village de la Sarthe, ensuite dans une banlieue parisienne. Dans cette cité inhospitalière des années 60, la jeune narratrice fait l'expérience amère et décevante du racisme, de l'injustice et de l'intolérance, vécus à l'école mais aussi par procuration, car sa grand-mère Julia, que la famille appelle Man Ya, y connaîtra toutes les facettes de l'exil'3. Pendant les six longues années passées en France, la grand-mère illettrée, tout comme le Petit Poucet de Perrault, se donnera comme mission de « marquer » le chemin vers la Guadeloupe pour ses petits-enfants, de les habituer aux sons du créole, aussi bien qu'aux saveurs de sa nourriture. Comme on le constate à la fin du récit, le retour dans l'île ne sera pas facile, mais la narratrice avoue être prête à surmonter toutes les difficultés pour s'y enraciner.

Le succès de ce récit de Pineau, attesté par le Prix Terre de France obtenu en novembre 1996, confirme le succès grandissant des textes biographiques, autobiographiques et autofictionnels. A titre d'exemple, citons le volume d'articles Récits de vie de l'Afrique et des Antilles (1998), 
témoignant de l'intérêt de la critique contemporaine pour ce genre de textes. Les trois axes thématiques de ce volume, Exil, Errance, Enracinement, soulèvent la problématique identitaire des récits de vie, intimement liée à l'espace postcolonial éclaté, voire pluriel. A l'éclatement de l'espace correspond l'éclatement des récits (auto)biographiques, composés d'une multiplicité d'histoires s'enchevêtrant selon les méandres de la mémoire des narrateurs qui les assument. Cette tendance vers la dérive textuelle et le brouillage des genres n'échappe pas à l'écrivain et théoricien martiniquais Edouard Glissant, fin observateur de la richesse littéraire des Antilles : « Les lecteurs », constate-t-il dans Traité $d u$ tout-monde, « aiment de plus en plus ces mélanges de genres, les romans qui sont des traités d'histoire, les biographies qui, sans cesser d'être exactes et minutieuses, s'apparentent à des romans » (174). Cette littérature, telle que pratiquée par Condé, Chamoiseau, Confiant, Pineau et Glissant, pour n'en citer que les noms les plus connus, a tendance à s'éloigner des genres canoniques, préférant les accumulations de récits parfois irréconciliables qui remettent en question le rapport fiction / réalité. Rappelons à ce propos le récit pour la jeunesse Un Papillon dans la cité, au sujet duquel Gisèle Pineau affirme que « [c]e n'est pas vraiment [son] histoire » («Causerie » 220). De plus, on constate que la thématique du récit (exil, déracinement, quête d'identité) revient dans la plupart des écrits de cet auteur. Se référant à un autre récit destiné à la jeunesse, Caraïbes sur Seine (1999), Pineau précise que s’il est vrai que la plupart des événements racontés sont fictionnels, il n'est pas moins vrai qu'elle y a inséré une histoire 4 qui l'a fait beaucoup souffrir quand elle était enfant ${ }^{5}$.

L'Exil selon Julia, classé par certains dans le genre autofictionnel (Bonnet 1997, Gyssels 1998), est certainement le texte le plus chargé d'éléments autobiographiques, fait attesté, d'ailleurs, par l'auteur elle-même, dans plusieurs prises de parole, ainsi que par la présentation du texte sur la quatrième de couverture ${ }^{6}$. Le discours paratextuel nous fournit donc un certain nombre d'éclaircissements qui, corroborés avec les expériences de la narratrice, nous permettent de les attribuer à l'auteur. A ce sujet, Thomas Spear note qu'une des caractéristiques de la nouvelle autobiographie antillaise, telle que pratiquée par Césaire, Zobel, Condé ou Pineau, c'est que «le pacte autobiographique n'est pas établi de façon concrète avec un nom de personnage de roman ou de poésie » (Spear 164), ce qui n'exclut pas la présence d'éléments biographiques vérifiables.

\section{Ici-là et là-bas ${ }^{7}$ : exil ou délivrance?}

Il est indéniable que L'Exil selon Julia contient de nombreux énoncés de type géographique et socio-historique sortant des souvenirs que Pineau a gardés de ses premières années de vie passées en France, en Afrique et en Guadeloupe. Le désir de raconter son enfance est doublé de l'intention avouée de dévoiler les blessures profondes de l'intolérance et du racisme, le plus souvent passées sous silence par les exilés en quête d'un espace plus accueillant. L'Exil selon Julia, précise Pineau, «n'est pas simplement une chronique familiale, c'est un livre sur la tolérance, sur les préjugés » (« Entretien » 1212). Dans un autre entretien elle ajoute : «j’ai vécu en France un exil par procuration auprès de ma grand-mère puisqu'elle, c'était vraiment une exilée » (« Causerie » 223).

Notons tout d'abord qu'en dépit de son caractère fragmenté, L'Exil selon Julia s'organise autour de l'impact de l'exil parisien sur la formation identitaire de la jeune narratrice. Le triple exil géographique, linguistique et racial, bien que vécu à travers des expériences différentes par l'enfant et par sa grand-mère, contribuera à la prise de conscience de leur aliénation profonde 
dans un ici-là urbain qui, de l'avis de Julia, est vidé de toute mémoire vivante. Précisons qu'au cours du récit ce déictique renvoie tour à tour à l'espace réel ou rêvé où évoluent les personnages, se chargeant de significations différentes selon leur état d'esprit, et surtout selon la distance qui les sépare de cet espace. A ce vocable créole fait pendant un là-bas également associé au lieu d'exil ou à l'espace insulaire. Ce dernier s'identifie souvent au Pays (à la Guadeloupe) vers lequel convergent les pensées nostalgiques des exilés.

D'entrée de jeu, on constate que pour beaucoup de jeunes femmes antillaises la France se présente comme un là-bas où elles espèrent être « sauvées du joug paternel, dégagées du sacerdoce d'aînesse, épargnées du destin des vieilles filles qui ne trouvent plus d'ivresse qu'en Dieu » (Exil 14-15). C'est le cas de Daisy, la mère de la narratrice, qui désire avoir une meilleure vie que celle de ses parents :

Parce qu'il est dit qu'on ne lit pas sur le front des personnes s'ils communient avec anges ou démons, elle ne cherche pas les fréquentations d'ici-là. Elle a vu comment l'homme qui monte haut, tombe et se casse dans une facilité de jouet. A présent, à cause de la faillite familiale ${ }^{8}$, elle sait aussi que les coquins ont souvent belle figure, sourires d'aristocrates, cravate et papiers à signer. (Exil 24-25)

Attirée par le mirage de l'ailleurs, Daisy accepte sans aucune hésitation de se marier avec Maréchal, un homme dont la peau n'est pas aussi claire que la sienne et qui, dans d'autres circonstances, aurait été difficilement accepté par sa famille. En écoutant les paroles de Maréchal, militaire en permission, Daisy se voit déjà parcourir le monde, loin de sa petite île qui n'a plus rien à lui offrir.

D'autres jeunes femmes, telle la mère de la narratrice Félicie, fuient l'île après avoir confié leur bébé à une vieille mère : "Man $\mathrm{Ya}^{9}$ raconte aux curieux que ma mère lui envoie chaque mois un gros mandat pour mon éducation. Elle $\mathrm{m}$ 'a appris à ne pas la contredire quand elle ment à ce sujet » (Papillon 7). Ce n'est que dix ans plus tard que la mère de Félicie, mariée en France, sera capable de faire venir sa fille, au désespoir de la grand-mère ${ }^{10}$. Par contre, Sybille, autre mère célibataire de L'Âme prêtée aux oiseaux, emmène son fils Marcello à Paris et l'élève toute seule, en lui cachant l'existence de son père jusqu'à l'âge de dix-sept ans. Elle travaille comme infirmière et réussit à offrir à son fils une vie décente mais, en dépit de toutes les précautions de la mère et de son amie Lila, celui-ci retournera en Guadeloupe pour rencontrer son père. Enfin, d'autres personnages féminins, tels Olga et Mina (Chair Piment), seront poursuivis par une malédiction familiale malgré la distance qui les sépare de leur île. Les raisons de leur exil intérieur ne seront dévoilées qu'à la fin du roman, lorsqu'on constate qu'on ne peut pleinement jouir du présent tant qu'on n'a pas réglé les affaires du passé ${ }^{11}$.

Afin de donner plus de crédibilité à l'urgence de quitter le pays d'origine frappé par la fatalité de l'Histoire, la narratrice de L'Exil selon Julia cède la parole aux grandes personnes qui ne cessent d'adresser une exhortation pathétique aux enfants du Pays :

[...] Enfants! Rien, il n'y a rien de bon pour vous au Pays, disaient les grandes personnes. Antan, ce fut une terre d'esclavage qui ne porte plus rien de bon. Ne demandez pas après ce temps passé! Profitez de la France! Profitez de votre chance de grandir ici-là! $\mathrm{Au}$ Pays, la marmaille parle patois. Profitez pour apprendre le 
français de France...Combien de Nègres vous envient, vous n'en avez pas idée. Y a tant de jalousie...C'est pas facile d'échapper à Misère, Malédiction et Sorcellerie, ces trois engeances du Mal qui gouvernent là-bas. (Exil 28)

Signalons en passant que Suzon Mignard donnera le même conseil à la jeune Mina, que sa demi-sœur Olga fera venir à Paris après la mort de ses parents et de sa petite sœur Rosalia : « Je te conseille pas de revenir ici-là, Mina...Reste tant que tu peux là-bas en France...Loin du Mal et de ses trente-deux mille tentacules...[...] Sauve-toi loin de Piment où il pousse rien que la haine et les fleurs du Mal» (Chair 199). Cependant, vingt ans plus tard, Mina constate que «[1] a Guadeloupe était partout. Elle n'avait jamais cessé d'être là » (Chair 131), malgré les efforts de la jeune femme d'oublier l'île et son passé douloureux. Même leitmotiv dans L'Âme prêtée aux oiseaux ; Néhémie, jeune femme capable d'interpréter les rêves, conseille à Marga d'aller en France si elle veut échapper à la fatalité antillaise : «Là-bas est ta fortune [...] Ici-là : le cimetière, là-bas: France-Délivrance » (Âme 60).

Bien que ces références à l'ici-là guadeloupéen soient chargées de clichés, on y reconnaît les motifs récurrents de la littérature antillaise : le lourd héritage de l'esclavage, la misère, la sorcellerie, la malédiction et la folie qui s'abat inexplicablement sur certains ${ }^{12}$. Pour échapper à ce Mal à la fois historique et métaphysique, bien des jeunes nourrissent l'espoir de s'épanouir loin de leur petite île et profitent de la première occasion pour s'en aller vers des endroits plus prometteurs. Néanmoins, lors des fêtes familiales passés avec des amis antillais, la narratrice de L'Exil selon Julia constate qu'en dépit des couleurs sombres utilisées pour dépeindre l'île d'origine, ceux qui avaient débarqué sur la terre promise ne pouvaient cacher les « reflets merveilleux » qui « scintillaient dans leurs yeux » au souvenir du Pays, devenu subitement objet de désir, dont on parlait « avec amour, nostalgie et dépit...» (Exil 29). Dans la petite cuisine de leur appartement parisien, elle entend sa mère partager son amertume et ses désillusions avec d'autres femmes de militaires, exilées elles aussi dans un pays qui, à les en croire, avait oublié le dévouement et les sacrifices de leurs maris : « Tout ici-là n'est que parade, grand arsenal de mots pour éblouir et ébranler [...] Pauvres vies de figurants de la nation [...], héros anonymes qui ont donné toute leur jeunesse à la France et n'ont connu qu' avec parcimonie le levain de la gloire » (Exil 13-14).

Comme on peut le constater, dans tous ces récits, le là-bas utopique perd souvent ses attraits après s'y être établi. Ce nouvel espace, devenu l'ici-là de tant d'Antillais, se présente comme un endroit froid et inhospitalier, où certains dépérissent lentement, telle la vieille Julia :

Mon Dieu, la froidure entre dans la chair et perce jusqu'aux os. Tous ces Blancs-là comprennent pas mon parler. Et cette façon qu'ils ont à me regarder comme si j'étais une créature sortie de la côte de Lucifer. Faut voir ça pour le croire. A mon retour en Guadeloupe, je raconterai à Léa que Là-Bas, la France, c'est un pays de désolation. (Exil 55)

Le confort et l'abondance matérielle n'impressionnent guère la vieille Antillaise : « Et je comprends pas pourquoi des Nègres vont se perdre dans ce pays-là » (Exil 65). Bien qu'elle s'épuise à faire toutes sortes de travaux ménagers, Julia rêve nuit et jour de son « chez soi » ensoleillé qui nourrissait son corps, et surtout son esprit. Ici, en France, sa famille croit qu'il suffit de la mettre à l'abri des soucis matériels et de l'abus de son mari pour qu'elle soit heureuse. Ils font semblant de ne pas voir que « on donne pas nourriture à son esprit» (Exil 65). Mais, ce qui rend la vieille 
Antillaise encore plus triste, c'est l'état des enfants de la cité, obligés à vivre dans un lieu stérile, coupé des sources vitales de la terre :

Quand elle retombe dans sa mélancolie, la cité lui apparaît comme une contrée déshéritée du savoir principal. Elle y voit croître toute l'ignorance du genre humain [...] Les enfants qui poussent là, dans la geôle de ces maisons en dur, perdent assurément le chemin du bon sens, à rôder qu'ils sont, si loin des essences de la vie, se dit-elle ... (Exil 128)

A force de les regarder s'aliéner dans le vide de l'espace parisien, Julia cherche un moyen qui lui permette de préparer le retour des plus jeunes de sa famille dans l'île de leurs ancêtres. Aussi, ne perd-elle aucune occasion de parler en créole des merveilles de son île, mais aussi de la cruauté de l'Histoire dont elle veut que ses petits-enfants se souviennent des années plus tard.

Le là-bas illusoire est également démystifié dans Un Papillon dans la cité. La narratrice Félicie, arrivée en France à l'âge de dix ans, constate le fossé entre le discours mensonger de la lettre de sa mère, calqué sur celui des images publicitaires, et la triste réalité perçue dans les récits d'autres insulaires attirés par le mirage d'une vie meilleure :

J'aurais dû prévoir que maman habitait dans un immeuble. Ceux qui parlaient de la France disaient que là-bas, les gens vivaient dans des kalòj [poulailler] à poules. Peut-être à cause de sa lettre où elle m'avait fait miroiter sa « vie meilleure », j'avais imaginé une vraie maison, du genre de celles photographiées dans Maisons Françaises [...], une vraie maison avec cheminée, toit de tuiles, fleurs au balcon, petite allée tapissée de gravillon. (Papillon 32)

Plus tard, Félicie observe elle aussi les enfants de son quartier, « aux yeux plus tristes que les cages d'escalier des bâtiments de la Cité » (Papillon 90). Lorsqu'elle fait miroiter devant les yeux de son meilleur ami Mohamed la beauté de la mer qu'il n'a jamais vue, l'esprit du petit Marocain saute « la muraille des immeubles gris de la Cité » (Papillon 91) afin de quitter la triste réalité du quotidien où il risque de s'engouffrer.

Mina, la protagoniste de Chair Piment, perçoit de la même façon la cité parisienne, décrite dans toute sa désolation physique et humaine, révélatrice de son exil intérieur :

De son septième étage, Mina découvrait toute la cité. Son univers depuis dix ans. Trois vieilles tours effarées taillées dans le béton. Cinq barres dressées entre des arbres maigres. Et six cubes couverts de tags. Derrière les façades bourgeonnantes d'antennes paraboliques, il y avait des appartements, cages à poules et clapiers à lapins, où vivaient les familles. Aussi haut que portait le regard, la cité hésitait entre ruine et loques. (Chair 21) ${ }^{13}$

A la différence de sa demi-sœur Olga, préoccupée à l'extrême de sa carrière, Mina se contente de travailler à la cantine d'un lycée parisien, où elle se lie d'amitié avec d'autres exilées. Rentrée chez elle, elle tue le temps avec des inconnus qui ne l'intéressent que pour le simple plaisir corporel, dans l'espoir d'oublier son passé douloureux, et surtout la mort de sa sœur Rosalia. En effet, le fantôme enflammé de Rosalia la suit partout, comme sa Guadeloupe natale qu'elle s'efforce d'enterrer dans l'oubli $^{14}$. Dans cet ici-là si éloigné de son lieu d'origine, Mina se donne l'illusion de se forger une identité fictive, de mettre un masque d'indifférence et de froideur pour cacher son âme écorchée et la protéger d'autres blessures : «Non, ici-là personne ne pourrait la trahir. C'était magique! Elle pouvait s'inventer une enfance sereine, se bâtir une vie sans tourment. Mentir et conter à l'infini. Personne ne saurait. Personne ne savait » (Chair 137). Cependant, ce n'est que son retour en Guadeloupe qui mettra fin à ses tourments, suite à la découverte de la malédiction 
qui pesait depuis longtemps sur sa famille. A ce sujet, Florence Ramond Jurney avance l'hypothèse que c'est grâce à la diversité de la communauté guadeloupéenne que les personnages principaux de Chair Piment réussissent à guérir. Il s'agirait d'une " guérison identitaire » (Jurney 33) qui ne pourrait se produire qu'après avoir configuré un espace identitaire capable de « faire le lien avec le passé » (Jurney 37). Pour Mina, cet espace prend forme grâce à une communauté de femmes exilées qui, par leurs bribes d'histoires, complètent son histoire à elle.

Une première conclusion s'impose : l'organisation et la qualité du nouvel espace constituent une source permanente de tristesse et d'aliénation pour les personnages de Pineau. Dans ces conditions, l'ici-là et là-bas entrent dans une relation antagoniste, trahissant les désillusions des Antillais découvrant une France inhospitalière, rongée par ses propres problèmes sociopolitiques. Afin d'expliquer l'état dépressif de la diaspora antillaise, mais aussi de l'Hexagone, Sam Haigh entreprend une lecture psychanalytique de L'Exil selon Julia de Pineau en se servant de deux textes de Julia Kristeva, Étrangers à nous-mêmes (1988) et Contre la dépression nationale (1998), ainsi que de l'étude The Melancholy of Race: Psychoanalysis, Assimilation, and Hidden Grief (2001) d'Anne Anlin Cheng. Dans son article intitulé « Migration and Melancholia: from Kristeva's 'Dépression nationale' to Pineau's 'Maladie de l'exil'»(2006), Haigh établit un lien entre la maladie de l'exil des personnages de Pineau et la « dépression nationale » des Français attribuée par Kristeva aux événements de la deuxième guerre mondiale, suivis par l'écroulement de leur empire colonial. Ainsi, de l'avis de Haigh, l'ingratitude de la France envers les Antillais constituet-elle le point de départ de l'état dépressif de Maréchal, le père militaire de la narratrice, tandis que l'éloignement de l'espace antillais et l'arrivée dans un espace froid et inhospitalier déclenchent la « mélancolie raciale » de Julia, éprouvée, selon Cheng, par les exilés de partout. L'espace urbain aliénant des banlieues parisiennes, preuve vivante de la double ségrégation sociale et raciale, démentit l'image mensongère de là-bas vers lequel aspirent tant de personnages de Pineau. Quant à Julia, qui subit un exil imposé par sa famille, elle souffre de claustrophobie dans cet espace coupé de la terre fertile de son île, qui alimentait son corps, et surtout son esprit. Il n'est donc pas étonnant que dans ce lieu de désespoir la plupart des personnages féminins de Pineau vivent en exil. La jeune narratrice de L'Exil selon Julia souffre principalement à cause de l'intolérance raciale à laquelle sa famille doit faire face dans le milieu parisien des années 60. Voici l'incipit du récit, chargé d'une violence verbale incompréhensible pour un enfant stigmatisé à cause de la couleur de sa peau :

\author{
Négro \\ Négresse à plateau \\ Blanche-Neige \\ Bamboula \\ Charbon \\ Et compagnie... (Exil 11)
}

La narratrice n'a que douze ans lorsqu'elle s'aperçoit des préjugés et du racisme de cet ici-là qui pourtant est son pays de naissance. Elle a du mal à comprendre pourquoi on lui répète de retourner chez elle, en Afrique : « Je veux bien retourner dans mon pays. Mais quel pays? Quelle Afrique? 
(Exil 139-40). Rappelons que ses parents avaient déjà fait l'expérience de l'Afrique, dont l'enfant ne garde que de vagues souvenirs. Comme tant d'autres Antillais, ils avaient constaté que la terre de leurs ancêtres ne les avait pas accueillis à bras ouverts, que ce détour n'arrivait pas à guérir la blessure originelle de l'esclavage et du déracinement ${ }^{15}$. Etablis en France, nouvelle déception : ils ne sont qu'une minorité trop visible pour les Français qui n'étaient pas habitués à la diversité raciale. Même une Antillaise à succès comme la speakerine martiniquaise Sylvette Cabrisseau se voit obligée de quitter son poste suite au mécontentement exprimé par des téléspectateurs xénophobes dans leurs lettres anonymes :

« Je ne suis pas raciste, mais cette Sylvette Cabrisseau ne parle pas un français correct et cela est dangereux pour la pureté de la langue française et nocif aux jeunes enfants qui apprennent à parler...»; ou bien encore : « Je n'ai rien contre les Noirs qui restent dans leur pays, mais la négresse qui présente les programmes fait peur à ma petite fille. Chacun chez soi! ». Et puis... « L'ORTF déshonore la France en affichant des bamboulas! Il y a suffisamment de belles Françaises dans nos provinces pour nous épargner cette laide figure... (Exil 102)

Ces commentaires témoignent de la difficulté de faire l'apprentissage de l'altérité même après le succès du mouvement de la Négritude. La peur et le rejet de l'Autre différent de Soi se manifestent non seulement au niveau individuel, mais aussi au niveau collectif.

Pour la narratrice de L'Exil, l'école représente l'endroit le plus chargé de racisme. A la différence de Félicie, qui a la chance d'avoir une institutrice désireuse d'ouvrir l'esprit de ses élèves vers le monde ${ }^{16}$, Marie se heurte au racisme excessif de sa méchante maîtresse, Madame Baron, qui l'oblige à rester sous son bureau pour ne pas voir « [s]a figure de négresse, [s]a peau noire » (Exil 152). Comme elle a honte de relater cet incident à ses parents, la jeune fille préfère le raconter dans une lettre envoyée à sa grand-mère : «Comment vivre dans un pays qui vous rejette à cause de la race, de la religion ou de la couleur de peau? » (Exil 153), écrit-elle à Man Ya. En outre, touchée par la lecture du journal d'Anne Franck, elle s'imagine écrire l'histoire de sa vie enfermée dans sa chambre pour ne pas être obligée de montrer sa peau noire à ceux qui n'aiment pas la voir. De surcroît, au souvenir des contes de Man Ya, elle rêve de se débarrasser de sa peau afin de se laisser emporter par les vents du Pays d'origine. La « délivrance » de la narratrice n'arrivera que grâce à la fermeture des écoles pendant les événements de mai 68. L'année suivante, la démission de Charles de Gaulle déterminera son père à quitter définitivement la France pour retourner en Guadeloupe, au grand contentement de sa fille.

\section{L'entre-deux}

En postulant le rapprochement des lieux éloignés, l'ici-là traduit le lien intime entre l'exilé antillais et son espace d'origine. La juxtaposition métaphorique de plusieurs endroits témoigne de sa capacité de vivre simultanément dans le réel et dans l'imaginaire. Ce dernier, espace essentiellement mémoriel, n'est autre que l'ici-là dont parle Glissant dans Poétique de la Relation 
et dans Traité $d u$ tout-monde, une des nombreuses traces qui ont facilité la survie de la population antillaise. Ainsi, le corps de Man Ya se trouve-t-il ici-là, en terre d'exil, mais « son esprit voyage sans fatiguer entre la France et son Pays Guadeloupe, où chaque jour elle espère retourner » (Exil 16). Au grand étonnement de ses petits-enfants, la grand-mère « est là, sans être là» (Exil 123). En observant le mal du pays ressenti d'une façon si douloureuse par la vieille Julia, la narratrice cherche à définir l'état de sa grand-mère : «C'est habiter Là-Bas, habité par le Pays » (Exil 121). Autrement dit, la distance entre la Guadeloupe et le lieu d'exil s'annule en esprit, seul capable de libérer Julia de l'emprise d'un ici-là aliénant. Le lointain se métamorphose en espace intérieur grâce à la magie du souvenir de son jardin créole qui efface toutes les douleurs passées. Valérie Loichot note à ce sujet que « Man Ya non seulement se souvient du pays, mais elle s'y enracine et le devient. Sa mémoire du pays est corporelle, charnelle, incarnée : c'est une mémoire des racines qui dépasse la binarité entre mémoire naturelle et mémoire culturelle » («Reconstruire dans l'exil » 29). Ajoutons à cela les commentaires de Dominique Licops au sujet du modèle biologique de l'épanouissement de l'identité en Guadeloupe, contrasté avec le modèle scriptural offert par les pays occidentaux. Rappelons à ce propos les leçons d'écriture que les enfants donnent à la grand-mère illettrée qui ne voit pas dans les lettres de son nom toute la richesse de couleurs de son identité créole. Un autre exemple hautement suggestif est l'épisode où Man Ya lave les lettres noires d'un cahier d'écolier de ses petits-enfants afin d'y faire place à leurs dessins colorés. Tous ces exemples mettent en évidence l'antagonisme des deux espaces perçus par Julia non seulement en termes géographiques, mais aussi en termes culturels. La civilisation essentiellement orale qui a façonné son esprit constitue, à ses yeux, un espace incompatible avec l'espace scriptural auquel ses petits-enfants essaient de l'initier. De plus, le souvenir des lettres mensongères que son mari Asdrubal lui avait envoyées en temps de guerre la blesse toujours. Elle préfère donc rester entre les deux espaces culturels, où elle attend le moment de son retour en Guadeloupe.

Félicie, la narratrice enfant d'Un Papillon dans la cité, vit aussi dans l'entre-deux, mais elle supporte mieux le mal physique et moral de l'exil : «Des fois, je pense à Laurine, aux belles vacances qu'elle doit vivre à Haute-Terre. Je songe à tous mes amis que j'ai laissés là-bas. Et mon cœur se serre » (Papillon 66). Bien que très jeune, cette narratrice n'associe pas ce joli « papillon » à une terre paradisiaque, car elle se rappelle bien la furie des cyclones annuels, la menace constante du volcan la Soufrière, ainsi que les tremblements de terre. " Pourtant », avoue-t-elle au lecteur, " il ne se passe pas un jour sans que je songe à ma vie là-bas » (Papillon 69).

Il est indéniable que la différence d'âge et d'expérience entre les deux personnages rend leur exil plus ou moins supportable. Avec Julia, Pineau attire l'attention sur le danger de l'exil imposé à un âge qui rend l'apprentissage d'un nouveau code culturel quasiment impossible, mais elle souligne aussi la capacité de la femme antillaise de résister à toutes les intempéries de la vie. Julia, tout comme Reine sans Nom, la grand-mère de Télumée du roman Pluie et vents sur Télumée Miracle de Simone Schwarz-Bart, attendra la fin de ses jours bien ancrée dans son jardin créole.

Quant à Mina, jeune femme malheureuse et inadaptée, même après des années passées à Paris elle ne peut s'empêcher de retrouver sa Guadeloupe natale dans tout ce qui l'entoure :

Suspendue aux cintres dans l'armoire. Mêlée aux fleurs du papier peint. Enfermée dans des pots de confiture de goyave et d'ananas. Au sec, parmi les écorces de cannelle et les noix de muscade, dans des boîtes en fer-blanc qui avaient autrefois contenu des biscuits au beurre frais de 
Normandie. Gardée dans la pulpe des jus d'orange et de pamplemousse. Serrée dans son cœur et sa mémoire. Dans les apparitions de Rosalia... (Chair 131)

La France où elle vit son exil douloureux n'arrive pas à gommer les parfums et les saveurs de l'espace domestique insulaire qu'elle s'efforce de refouler par tous les moyens ${ }^{17}$. Nombreuses sont d'ailleurs les références à la cuisine antillaise non seulement dans les romans de Pineau, mais aussi dans ceux de Maryse Condé, de Simone Schwarz-Bart, de Patrick Chamoiseau et d'autres. Il suffit de mentionner Un Plat de porc aux bananes vertes (1967) d'André et de Simone Schwarz-Bart et le dernier roman de Condé, Victoire, les saveurs et les mots (2006).

De ce qui précède, une autre conclusion s'impose : pour certains personnages de Pineau, le souvenir de la terre natale jette un pont entre l'ici-là vu comme lieu d'exil et le là-bas remémoré comme espace vital indispensable à leur épanouissement harmonieux. Pour d'autres, qui possèdent peu de souvenirs de leur lieu d'origine, ou qui l'imaginent tel qu'esquissé dans les récits d'une grand-mère, cet espace interstitiel se matérialise en un récit de vie hybride. Prenons l'exemple de la narratrice de L'Exil selon Julia, née en France de parents antillais, qui fera son premier voyage en Guadeloupe à l'âge de cinq ans. C'est à cette occasion qu'elle rencontrera ses grands-parents et qu'elle découvrira avec enchantement les merveilles de l'île. Cependant, retournée à Paris, sans la présence de sa grand-mère paternelle au sein de sa famille, la narratrice n'aurait pas eu la chance de se bâtir une mémoire généalogique capable de la préparer en vue du retour définitif en Guadeloupe. En effet, la parole créole de Man Ya recrée tous les soirs l'espace insulaire avec tout ce qu'il a de contradictoire et de magique. Ses histoires vraies de la traite et de l'esclavage, ainsi que les vieux contes animaliers, font resurgir devant les yeux de ses petits-enfants le monde magico-religieux de son île chérie. A la fin de son séjour forcé en France, l'aïeule a la certitude de les avoir suffisamment préparés pour leur retour sur l'île.

Après le départ de Julia, la jeune fille se réfugie dans l'écriture, seule consolation de l'exil qu'elle ressent avec de plus en plus d'acuité : «Quand je suis dans mes écritures, que personne n'a le droit de lire, on me laisse tranquille. Les élèves de ma classe me trouvent soudain intéressante. Ils n'en reviennent pas que la seule négresse-bamboula d'Afrique de la classe les surpasse dans leur belle langue de France » (Exil 159). Des lettres informatives qu'elle adresse à Man Ya, la jeune fille passe au journal intime, témoin de sa passion grandissante pour l'écriture : "Finalement, je me rends compte que je ne t'envoie plus du tout de courrier. Je suis une copieuse. J'imite Anne Franck et j'écris à un cahier » (Exil 156). Affligée à cause du racisme dont elle est la cible à l'école, mais aussi à cause de l'inquiétude de la famille face aux événements politiques précédant la démission du général de Gaulle, la narratrice se sent de plus en plus attirée par Anne Franck, dont le Journal constitue un des intertextes privilégiés de Pineau. Cependant, les bruits des événements tumultueux de mai 68 se font aussi entendre à travers les lignes du journal où la jeune fille réfléchit à l'impact de la destinée de Charles de Gaulle sur les décisions de vie de ses parents : « Si papa n'était pas entré en dissidence pour le rejoindre, où serions-nous à l'heure qu'il est? Si papa n'avait pas porté l'uniforme de l'armée française, ma maman Daisy lui aurait-elle dit oui pour la vie? Voilà comment des Antillais naissent en France » (Exil 161). Aussi, la narratrice se forge-t-elle un nouvel espace situé entre l'ici-là parisien et le là-bas insulaire, entre le réel et l'imaginaire. Ce «troisième espace », comme l'appelle Homi Bhabha dans The Location of Culture, c'est un espace intermédiaire qui facilite la construction identitaire de l'enfant à partir des expériences 
douloureuses vécues à l'école, mais aussi à partir des histoires de la grand-mère. Au moment où l'on décide la date du départ définitif de France, Marie, qui vient d'avoir treize ans, s'exclame : « Je me ferai papier, encre et porte-plume pour entrer dans la chair du Pays» (Exil 168). Cependant, elle ne peut s'empêcher de s'inquiéter sur l'accueil que le Pays de leurs parents fera à des enfants nés en métropole : « Ce Pays qui bat et saute comme un cœur, là-bas. Est-ce qu'il nous reconnaîtra comme ses enfants? » (Exil 169-70).

Cette période de tourments et d'épanouissement créatif aidera la narratrice à mieux se comprendre, mais aussi à se préparer pour le voyage de retour. Cette fois-ci, c'est elle qui s'identifiera au petit Poucet des contes de son enfance : « J'ai perdu les cailloux de mon Pays, mais j'y retourne. Man Ya a marqué le chemin. Et je suis comme Poucet » (Exil 173-74). En effet, au moment du débarquement à la Martinique ${ }^{18}$, la jeune fille constate avec surprise que « [1]es lieux ne sont pas étrangers. Tout ici-là est inconnu et pourtant reconnu [...] Tout ici-là est ami et ennemi [...] Tout ici-là est étonnement, tant misère et grandeur, beauté et laideur s'entrecroisent, s'imbriquent et se chevauchent » (Exil 177). La parole de la grand-mère et le mal de l'exil ont fait pousser des fleurs que la narratrice sera prête à cueillir.

Il s'ensuit que cet entre-deux insaisissable, espace configuré à la croisée de contradictions baudelairiennes, n'en reste pas moins un espace qui facilite la construction de l'identité relationnelle, telle que rêvée par la nouvelle génération d'écrivains de la Caraïbe. C'est justement ce lieu difficile à appréhender dans toute sa complexité que la narratrice de L'Exil selon Julia se propose de figer sur la page écrite. A la différence de sa grand-mère, qui ne fait plus confiance à l'écriture menteuse, sans mémoire et sans vie, la jeune fille s'y adonne avec plaisir. Le seul défi qu'elle entrevoit dans son entreprise scripturale, c'est de ne pas trahir l'héritage culturel légué oralement par Man Ya :

Comment démêler les rêves de la réalité? L'invention du véritable? Le réel du conte? [...] Craindre que ces îles modelées au lointain ne soient que construction de carton-pâte, décor de Cinémascope, mornes peints à la gouache pour colorer l'exil. Craindre et imaginer que tout n'ait été inventé par Man Ya. Se figurer que l'esclavage, Schœlcher, les Nègres marrons n'aient été que des acteurs mis en scène par Man Ya, juste pour nous donner une fierté, une histoire, une existence, un pays à aimer. (Exil 169)

La réappropriation de l'espace antillais par la jeune narratrice se fera donc dans un premier temps par le biais de l'écriture poursuivie dans un ici-là aliénant, auquel elle se soustrait en rêvant d'un là-bas porteur de l'espoir de délivrance ${ }^{19}$. Dans un deuxième temps, après son arrivée à la Martinique, la narratrice partira à la conquête de ce là-bas qui se métamorphose peu à peu en un ici-là exploré dans tous les sens, jusqu'à ce qu'elle en prenne complète possession. Son seul regret, c'est qu'elle grandit trop vite, ce qui lui laisse peu de temps pour jouir d'une enfance antillaise. Pourtant, les lectures et les expériences de son enfance parisienne, greffées sur les histoires vraies et imaginaires de sa grand-mère antillaise, lui faciliteront la prise de conscience de sa double appartenance culturelle. A l'instar d'autres personnages féminins de Pineau, la narratrice de L'Exil selon Julia n'a pas peur d'explorer « des lieux de mémoire fragiles » (Bonnet 174) afin de mener à bien sa quête identitaire. 


\section{Conclusion}

Au cours de cette analyse, nous espérons avoir démontré que pour les héroïnes de Pineau, la remémoration et l'écriture se présentent comme des espaces privilégiés, témoignant du difficile processus de construction identitaire de la femme antillaise qui a fait l'expérience de l'errance et du métissage culturel. Quant à la langue choisie pour ses écrits (auto)biographiques, Pineau suit l'exemple de sa génération d'écrivains antillais qui infusent le français de la langue de leur ethnicité dont l'usage ne devrait jamais devenir une contrainte : «C'est un français qui se nourrit de la poétique, des couleurs, de l'ambiance et des rythmes de la langue créole » ("Causerie » 226). En deuxième lieu, bien qu'associée souvent au mouvement des Créolistes, Gisèle Pineau semble ouvrir l'espace de son écriture vers le tout-monde glissentien, où chaque lieu est à la fois centre et périphérie : «Le nombril de ce monde se trouve partout et nulle part » (Exil 105), constate la narratrice de L'Exil selon Julia, fascinée, comme sa grand-mère par l'avalanche d'images médiatiques. Comme tant d'autres écrivains francophones contemporains, Pineau propose de dépasser le stade de la simple dénonciation du dualisme colonisateur / colonisé. A son avis, les nouvelles réalités du monde postcolonial obligent l'écrivain antillais à réfléchir plus profondément au sujet de l'identité relationnelle et du processus créateur originé non seulement dans le vécu personnel, mais aussi dans celui des autres. Ainsi, dans L'Âme prêtée aux oiseaux, l'auteur détruitelle symboliquement les barreaux des cages qui enferment les oiseaux porteurs d'amour, en ouvrant largement leurs portes pour qu'ils s'envolent vers l'espace de leur choix. Par ses écrits et ses prises de paroles, Gisèle Pineau se joint à d'autres auteurs antillais qui annoncent la culture pluraliste de l'avenir où l'on devrait mieux valoriser le métissage et les identités hybrides.

\section{Bibliographie-Corpus}

Pineau, Gisèle. Un Papillon dans la cité (1992). Paris : Éditions Sépia.

----. L'Exil selon Julia (1996). Paris : Éditions Stock.

-----. L'Âme prêtée aux oiseaux (1998). Paris : Éditions Stock.

-----. Chair Piment (2002). Paris : Mercure de France.

\section{Bibliographie - sources théoriques et critiques}

Belugue, Geneviève. « Entre ombre et lumière, l'écriture engagée de Gisèle Pineau ». Notre Librairie : Revue des Littératures du Sud 138-39 (1999-2000): 84-90.

Bhabha, Homi. The Location of Culture. London and New York : Routledge, 1994.

Bonnet, Véronique. De l'exil à l'errance: écriture et quête d'appartenance dans la littérature contemporaine des petites Antilles anglophones et francophones, thèse de doctorat, non publiée, Université Paris Nord, Paris XIII.

-----. « Gisèle Pineau : L'âme prêtée à l'écriture ». Notre Librairie : Revue des Littératures du Sud 138-39 (1999-2000): 91-97.

Crosta, Suzanne (dir.). Récits de vie de l'Afrique et des Antilles. Québec: Université Laval, GRELCA, 1998.

Gallagher, Mary (ed. and introd.). Ici-Là: Place and Displacement in Caribbean Writing in French. 
Rodopi, Amsterdam, 2003.

Githire, Njeri. «Horizons Adrift: Women in Exile, at Home, and Abroad in Gisèle Pineau's Works». Research in African Literatures, vol. 36, no. 1 (Spring 2005): 74-90.

Glissant, Édouard. La Poétique de la relation. Paris : Gallimard, 1990.

-----. Traité du tout-monde. Paris : Gallimard, 1997.

Gyssels, Kathleen. «L'exil selon Pineau, récit de vie et autobiographie ». Récits de vie de l'Afrique et des Antilles (dir. Suzanne Crosta), Québec: Université Laval, GRELCA, 1998, pp. 169187.

Haigh, Sam. «Migration and Melancholia: from Kristeva's 'Dépression nationale' to Pineau's 'Maladie de l'exil'». French Studies, vol. 60, no. 2 (2006): 232-50.

Hellerstein, Nina. "Violence, Mythe et Destin dans l'Univers Antillais de Gisèle Pineau». LittéRéalité vol.10, no 1 (1998): 47-58.

Jurney, Florence Ramond. «Transgresser l'insularité : Inscriptions de l'espace antillais dans Chair Piment de Gisèle Pineau. » LittéRéalité vol. XVI, no 1 (printemps/été 2004): 31-43.

Licops, Dominique. «Origi/nation and Narration: Identity as Epanouissement in Gisèle Pineau's Exil selon Julia». MaComère 2 (1999): 80-95.

Loichot, Valérie. « Reconstruire dans l'exil: la nourriture créatrice chez Gisèle Pineau ». Etudes Francophones vol.17, no. 2 (automne 2002): 25-44.

Mackward Christiane et Njeri Githire. " Gisèle Pineau : Causerie à Penn State (avril 2001) ». Women in French Studies (2001): 220-233.

-----. « Entretien avec Gisèle Pineau ». The French Review vol. 76, no 6 (May 2003): 1202-1215.

Maunier, Priscilla. "Odyssey of a double consciousness: commonalities and disjunctions in contemporary French Caribbean and Réunionese novels». International Journal of Francophone Studies, vol. 8, no. 2 (2005): 165-81.

Mugnier, Françoise. « La France dans l'œuvre de Gisèle Pineau ». Etudes francophones vol.15, no.1 (2000): 61-73.

Pineau, Gisèle. « Écrire en tant que Noire ». Penser la créolité, sous la direction de Maryse Condé et Madeleine Cottenet-Hage, Paris : Karthala, 1995, pp. 289-295.

Pineau, Gisèle et Marie Abraham. Femmes des Antilles. Trace et voix. Paris : Éditions Stock, 1998.

Spear, Thomas. «L'Enfant créole : la nouvelle autobiographie antillaise ». Récits de vie de l'Afrique et des Antilles (dir. Suzanne Crosta). Québec : Université Laval, GRELCA, 1998, pp. 143167.

Suárez, Lucia. «Gisèle Pineau: Writing the Dimensions of Migration». World Literature Today, vol. 75, nos. 3 /4 (Summer 2001): 9-21.

Vitiello, Joëlle. « Le corps de l'île dans les écrits de Gisèle Pineau ». Elles écrivent des Antilles, Paris : L'Harmattan, 1997, pp. 243-63.

\section{Notes}

${ }^{1}$ A ce propos, dans l'ouvrage Femmes des Antilles : traces et voix cent cinquante ans après l'abolition de l'esclavage (1998), réalisé en collaboration avec Marie Abraham, elle exprime clairement son intention de donner la parole aux femmes, dont la voix se fait rarement entendre dans l'espace public, bien qu'elles soient depuis longtemps le 
poteau-mitan de la famille antillaise. Pineau justifie sa prédilection pour les personnages féminins par l'importance primordiale que les femmes ont eue dans le processus de métissage des îles des Caraïbes : «C'est bien par le corps de la femme qu'est passé le métissage dans toutes les Antilles » («Causerie » 232), affirme-t-elle, tout en soulignant sa position féministe qui veut que le monde ne reproduise plus les modèles de victimisation de la femme. D'ailleurs, il suffit de penser aux romans La grande drive des esprits (1993), L'Espérance-macadam (1995) et Chair Piment (2002) pour nous rendre compte de l'étendue de la souffrance féminine, plus particulièrement de l'inceste auquel Pineau est parmi les premiers à s'attaquer. Cependant, il n'en demeure pas moins que l'auteur ne perd aucune occasion d'exprimer son optimisme au sujet du devenir de l'humanité :

Je perçois le monde comme une quête entre la souffrance et l'espérance. C'est une forme de recherche : dire la douleur comme pour l'éradiquer, ouvrir l'horizon sur le rêve, sur l'espérance d'une évasion possible de toutes les souffrances, de tous les passés douloureux. Celui des Antilles, certes, mais aussi celui des destinées individuelles d'ici et d'ailleurs. (« Entre ombre et lumière » 85)

${ }^{2}$ L'autre prénom de Gisèle Pineau.

${ }^{3}$ Lors de son premier congé en Guadeloupe, le père de la narratrice, militaire de profession, avait décidé d'emmener sa mère Julia en France, pour la soustraire à la violence de son mari Asdrubal.

${ }^{4}$ Il s'agit d'une idylle par correspondance entre l'héroïne et un jeune Américain qui ne savait pas qu'elle n'était pas blanche. Lorsqu'il a reçu la photo de la jeune fille, il a arrêté de lui écrire, ce qui a profondément blessé la narratrice de cette histoire.

5 Il n'est pas sans intérêt de mentionner qu'après la parution de son roman Histoire de la femme cannibale (2003), Maryse Condé adopte la même position : « J'ai voulu mêler directement mes expériences au cheminement de cette héroïne qui est un peu moi, mais n'est pas moi » (Voir du 3 avril 2003)

${ }^{6}$ « Gisèle a une alliée : Julia, dite Man Ya, la grand-mère venue en France pour fuir les brutalités de son mari. »

${ }^{7}$ Dans toutes les citations contenant ces deux vocables nous allons les mettre en évidence par des italiques.

${ }^{8}$ La famille de Daisy avait perdu tous ses biens à cause d'un prêt que le père avait signé à un faux ami qui, par la suite, avait mis en scène sa mort pour s'établir en France avec l'argent volé.

${ }^{9}$ La grand-mère de Félicie porte le même nom que celle de la narratrice de L'Exil selon Julia, ce qui atteste une fois de plus la présence d'éléments autobiographiques dans les deux textes. A ce sujet, Véronique Bonnet remarque le fait que « la fiction précède l'autofiction, frayant un chemin en direction d'une écriture de plus en plus autoréférentielle, de plus en plus impliquée dans le réel » (Bonnet, p. 196). Elle place ce récit «à la croisée de l'auatobiographie, des mémoires et de la fiction » (Bonnet 192).

${ }^{10}$ Notons à ce sujet que cette relation très proche entre une jeune protagoniste et sa grand-mère se retrouve aussi dans d'autres romans antillais, dont le bien connu Pluie et vents sur Télumée Miracle de Simone Schwarz-Bart. A la différence de Félicie, qui se sépare de Man Ya au début de l'adolescence, Télumée rejoint sa grand-mère Reine sans Nom vers le même âge. Dans les deux cas, la grand-mère est une source inépuisable d'amour et de sagesse.

${ }^{11}$ Gisèle Pineau n'est pas le seul auteur antillais à toucher à ce sujet. Les problèmes irrésolus du passé expliquent, par exemple, le retour de " l'étranger » Francis Sancher sur l'île où l'un des ses ancêtres avait commis un crime impardonnable (Condé, Traversée de la mangrove). Par ailleurs, presque tous les personnages errants des derniers romans de Maryse Condé essaient de régler certaines affaires de leur passé chargé, ou au moins d'en accepter les conséquences.

12 Simone Schwarz-Bart, avec qui Gisèle Pineau a beaucoup d'affinités, parlera de la même fatalité dans les termes suivants : «Lorsque, durant les longs jours bleus et chauds, la folie antillaise se met à tournoyer dans l'air au-dessus des bourgs, des mornes et des plateaux, une angoisse s'empare des hommes à l'idée de la fatalité qui plane au-dessus d'eux, s'apprêtant à fondre sur l'un ou l'autre, à la manière d'un oiseau de proie, sans qu'il puisse offrir la moindre résistance » (Pluie et vent sur Télumée Miracle 40).

13 « La logique de l'organisation de l'espace des migrants relève de l'emboîtement et de l'opposition horizontalité / verticalité » (Bonnet 232). 
${ }^{14}$ Florence Ramond Jurney parle des vides physique et culturel, ainsi que du vide des origines vécus par l'Antillaise Mina, mais aussi par le Français Victor (« Transgresser l'insularité : Inscriptions de l'espace dans Chair Piment de Gisèle Pineau »).

${ }^{15}$ Hérémakonon de Maryse Condé, ainsi que Juletane de Myriam Warner-Vieyra illustrent également l'échec du détour effectué par Véronica et Juletane afin de découvrir leurs racines africaines.

${ }^{16}$ Cette jeune institutrice organise une excursion en Guadeloupe dans le but d'offrir à ses élèves l'occasion de connâ̂tre un territoire de la France ignoré par la plupart d'entre eux. A cette occasion, Félicie passera quelques jours avec sa grand-mère Man Ya, tandis que son ami marocain Mohamed se réjouira de voir la mer pour la première fois. Lucia Suárez voit dans cette initiative « la possibilité de retourner à l'île d'origine grâce à un système d'éducation libéral et ouvert à tout le monde » (« Gisèle Pineau: Writing the Dimensions of Migrations », p. 13, notre traduction)

${ }^{17} \mathrm{Au}$ sujet de la reconstruction culinaire du pays, voir l'article de Valérie Loichot, « Reconstruire dans l'exil : la nourriture créatrice chez Gisèle Pineau », Études francophones 17 : 2 (Automne 2002) : 25-44.

${ }^{18}$ Avant de s'établir en Gouadeloupe, la famille doit faire un séjour à la Martinique, lieu de transition et d'adaptation décrit avec sensibilité et humour par la jeune narratrice.

${ }^{19}$ A la fin du récit Un Papillon dans la cité, la narratrice avoue à son copain Mohamed sa passion secrète : « J'écris. J'écris tout ce qui m'arrive...J'ai un cahier pour ça » (124). 